\title{
ADDITIVE MAPPINGS ON RINGS OF HOLOMORPHIC FUNCTIONS
}

\author{
R. B. BURCKEL AND SADAHIRO SAEKI
}

\begin{abstract}
Additive maps $T$ between rings of holomorphic functions, which satisfy the multiplier-like condition $T(z f)=\phi T(f)$ for some $\phi$ and all $f$, are characterized. No linearity or continuity assumptions are made. Earlier results by L. Bers on homomorphisms and by J. Becker on derivations are corollaries.
\end{abstract}

1. Introduction and notation. Throughout this paper $U, W, \Omega$ are nonvoid open subsets of the complex plane $\mathbf{C}$, and $\mathbf{Q}$ denotes the rational numbers, $\mathbf{R}$ the real numbers. By $C(U), H(U), H^{\infty}(U)$ we denote the sets of all continuous, holomorphic and bounded holomorphic functions on $U$, respectively. The identity function on $U$ is denoted by $I$, that is, $I(u)=u$ for all $u \in U$. It is convenient to designate with the same symbol, a complex number and the constant function with that value on whatever domain is under discussion. Additive mappings $T: H(U) \rightarrow H(W)$ which satisfy $T(I f)=\phi T(f)$ for all $f \in H(U)$ and some $\phi \in C(W)$ will be investigated. Their structure is completely determined and from this, various results about the structure of ring homomorphisms and derivations on $H(U)$ are recovered. See $\S 4$ below.

2. A key lemma. In the aforementioned theorems about ring homomorphisms and derivations much of the effort in known proofs goes into showing that the map is complex-linear or conjugate complex-linear. For example, formal power series are used to this end in Becker (1978). We will accomplish this with the following very elementary

Lemma. Let $B$ be a countable subset of $\mathbf{C}, Q$ a dense subset of $\mathbf{C}$. Let $c \in \mathbf{C}$ and $a \in \mathbf{C} \backslash B$. Then there is an entire function $g$ which satisfies $g(B) \subset Q$ and $g(a)=c$.

Proof. Let $b_{1}, b_{2}, b_{3}, \ldots$ be a one-to-one enumeration of $B$ and set $b_{0}=a$. Since $Q$ is dense, we can select $c_{1} \in \mathbf{C}$ so that $c+c_{1}\left(b_{1}-b_{0}\right) \in Q$. Then define $P_{1}(z)=c$ $+c_{1}\left(z-b_{0}\right)$. After polynomials $P_{1}, \ldots, P_{n-1}(n \geqslant 2)$ have been constructed, we can find $c_{n} \in \mathbf{C}$ so small that

$$
\sup \left\{\left|c_{n} \prod_{k=0}^{n-1}\left(z-b_{k}\right)\right|:|z| \leqslant n\right\}<2^{-n}
$$

Received by the editors January 24, 1983.

1980 Mathematics Subject Classification. Primary 30H05, 46E25.

Key words and phrases. Additive maps, rings of holomorphic functions, derivations, homomorphisms. 
and such that

$$
P_{n-1}\left(b_{n}\right)+c_{n} \prod_{k=0}^{n-1}\left(b_{n}-b_{k}\right) \in Q \text {. }
$$

Then define

$$
P_{n}(z)=P_{n-1}(z)+c_{n} \prod_{k=0}^{n-1}\left(z-b_{k}\right) \text {. }
$$

From these definitions and (1) we see that $g(z)=\lim _{n \rightarrow \infty} P_{n}(z)$ exists uniformly on every bounded subset of $\mathbf{C}$. For all $n \geqslant 1$ we have $P_{n}\left(b_{n}\right) \in Q$ [by (2), (3)], $g\left(b_{n}\right)=P_{n}\left(b_{n}\right)$ and $P_{n}\left(b_{0}\right)=c$.

REMARKS. This lemma occurs in Stäckel (1895) but may predate that paper. More recent refinements of it, which are of interest but are not needed for our subsequent development, are to be found in Maurer (1967), Barth and Schneider (1971/72), Sato and Rankin (1974) and Nienhuys and Thiemann (1976).

\section{The main results.}

Principal TheORem. Let $U, W$ be nonvoid open subsets of $\mathbf{C}, \phi: W \rightarrow \mathbf{C} a$ continuous, open map and $T: H(U) \rightarrow C(W)$ a map which satisfies

(1) $T$ is additive,

(2) $T($ if $)=i T(f) \forall f \in H(U)$,

(3) $T($ If $)=\phi T(f) \forall f \in H(U)$.

Then for each $f \in H(U)$,

$$
T(f)= \begin{cases}(f \circ \phi) T(1) & \text { on } W \cap \phi^{-1}(U), \\ 0 & \text { on a neighborhood of } W \backslash \phi^{-1}(U) .\end{cases}
$$

Proof. Let $N$ denote the set of all complex numbers $c$ such that $T(c f)=c T(f)$ for all $f \in H(U)$. It follows from (1) and (2) that $\mathbf{Q}+i \mathbf{Q} \subset N$, so $N$ is dense in $\mathbf{C}$.

Suppose $\phi^{-1}(U) \neq \varnothing$. Consider any $w \in \phi^{-1}(U \cap N), f \in H(U)$. For appropriate $h \in H(U)$ we have

$$
f(u)-f(\phi(w))=[u-\phi(w)] h(u) \quad \forall u \in U .
$$

Apply $T$, using (3) and the fact $\phi(w) \in N$ :

$$
T(f)-T[f(\phi(w))]=[\phi-\phi(w)] T(h) .
$$

Evaluate at $w$ to get

$$
T(f)(w)=T[f(\phi(w))](w), \quad f \in H(U), w \in \phi^{-1}(U \cap N) .
$$

Now consider any $c \in \mathbf{C}, w \in \phi^{-1}(U \cap N)$. Since $\phi$ is an open map, $\phi^{-1}(N)$ is dense in the open set $\phi^{-1}(U \backslash\{\phi(w)\})$, so there exist $w_{n} \in \phi^{-1}(U \backslash\{\phi(w)\}) \cap \phi^{-1}(N)$ with $w_{n} \rightarrow w$. The lemma furnishes an entire function $g$ such that $g\left(\phi\left(w_{n}\right)\right) \in c+N$, say, 
$g\left(\phi\left(w_{n}\right)\right)=c-q_{n}$, for all $n$, and $g(\phi(w))=0$. Then we have

$$
\begin{aligned}
0 & =T[g(\phi(w)) f(\phi(w))](w) \stackrel{(4)}{=} T(g f)(w)=\lim _{n \rightarrow \infty} T(g f)\left(w_{n}\right) \\
& \stackrel{(4)}{=} \lim _{n \rightarrow \infty} T\left[(g f)\left(\phi\left(w_{n}\right)\right)\right]\left(w_{n}\right)=\lim _{n \rightarrow \infty} T\left[c f\left(\phi\left(w_{n}\right)\right)-q_{n} f\left(\phi\left(w_{n}\right)\right)\right]\left(w_{n}\right) \\
& =\lim _{n \rightarrow \infty}\left\{T\left[c f\left(\phi\left(w_{n}\right)\right)\right]\left(w_{n}\right)-q_{n} T\left[f\left(\phi\left(w_{n}\right)\right)\right]\left(w_{n}\right)\right\} \\
& \stackrel{(4)}{=} \lim _{n \rightarrow \infty}\left\{T(c f)\left(w_{n}\right)-q_{n} T(f)\left(w_{n}\right)\right\}=T(c f)\left(w^{\prime}\right)-c T(f)(w) .
\end{aligned}
$$

This proves that $T(c f)(w)=c T(f)\left(u^{\prime}\right)$ for all $w^{\prime} \in \phi^{-1}(U) \cap \phi^{-1}(N)$. Since this set is dense in $\phi^{-1}(U)$, we get by continuity

$$
T(c f)(w)=c T(f)\left(w^{*}\right) \quad \forall w^{\prime} \in \phi^{-1}(U), f \in H(U), c \in \mathbf{C} .
$$

In particular,

$$
T(c)(w)=c T(1)(w) \quad \forall w \in \phi^{-1}(U), c \in \mathbf{C} .
$$

Given $f \in H(U), w \in \phi^{-1}(U)$, put (5) with $c=f\left(\phi\left(w^{\prime}\right)\right)$ into the right side of (4) to get

$$
T(f)(w)=f\left(\phi\left(w^{\prime}\right)\right) T(1)\left(w^{\prime}\right) \quad \forall f \in H(U), w \in \phi^{-1}(U) .
$$

Next suppose $\left[W \backslash \phi^{-1}(U)\right]^{\circ} \neq \varnothing$, where $\circ$ denotes interior. Consider $w \in$ $\left[W \backslash \phi^{-1}(U)\right]^{\circ} \cap \phi^{-1}(N), f \in H(U)$. Then $f(u) /\left(u-\phi\left(w^{\prime}\right)\right)=h(u) \in H(U)$. Apply $T$ to $f=[I-\phi(w)] h$, remembering that $\phi(u) \in N$, to get $T(f)=[\phi-\phi(w)] T(h)$, $T(f)(w)=0$. Since $\phi$ is an open map, such $w$ are dense in $\left[W \backslash \phi^{-1}(U)\right]^{\circ}$ and so we have

$$
T(f)(w)=0 \quad \forall f \in H(U), w \in\left[W \backslash \phi^{-1}(U)\right]^{\circ} .
$$

Next suppose that $w \in W \cap \partial \phi^{-1}(U), f \in H(U)$ and that $T(f)$ is not identically 0 in any neighborhood of $w$. Then there exist $w_{n} \in \phi^{-1}(U)$ such that $w_{n} \rightarrow w$ but $T(f)\left(w_{n}\right) \neq 0$. We have $\phi\left(w_{n}\right) \neq \phi\left(w^{\prime}\right)$ for all $n$ and, since $\phi$ is continuous at $w$, $\phi\left(w_{n}\right) \rightarrow \phi(w)$. Therefore Mittag-Leffler's theorem furnishes an entire function $h$ such that

$$
\left|h\left(\frac{1}{\phi\left(w_{n}\right)-\phi(w)}\right)\right|>\frac{n}{\left|T f\left(w_{n}\right)\right|} \quad \forall n .
$$

Noting that $w \in W \backslash \phi^{-1}(U)$, so $\phi(w) \in \mathbf{C} \backslash U$, we can define $g \in H(U)$ by

$$
g(u)=h\left(\frac{1}{u-\phi(w)}\right), \quad u \in U
$$

Then we have, by two applications of $(4)^{\prime}$,

$$
\begin{gathered}
T(f g)\left(w_{n}\right)=g\left(\phi\left(w_{n}\right)\right) f\left(\phi\left(w_{n}\right)\right) T(1)\left(w_{n}\right)=g\left(\phi\left(w_{n}\right)\right) T(f)\left(w_{n}\right) \\
\stackrel{(8)}{=} h\left(\frac{1}{\phi\left(w_{n}\right)-\phi(w)}\right) T(f)\left(w_{n}\right) \quad \forall n .
\end{gathered}
$$


It follows from (7) that $\left|T(f g)\left(w_{n}\right)\right|>n$ for all $n$. Since $w_{n} \rightarrow w \in W$ and $T(f g) \in$ $C(W)$, we have a contradiction.

This reductio ad absurdum proves that for each $f \in H(U)$ there is an open neighborhood of $W \cap \partial \phi^{-1}(U)$ in which $T f=0$. Uniting this neighborhood with the open set $\left[W \backslash \phi^{-1}(U)\right]^{\circ}$ and recalling (6), gives an open neighborhood $W_{f}$ of $W \backslash \phi^{-1}(U)$ such that $T f=0$ in $W_{f}$. Together with (4)' this completes the proof of the theorem.

Scholium. Let $U, W$ be nonvoid open subsets of $\mathbf{C}, \phi: W \rightarrow \mathbf{C}$ a continuous open map and $T: H(U) \rightarrow C(W)$ a map which satisfies

(1) $T$ is additive,

(2) $T($ if $)=-i T(f) \forall f \in H(U)$,

(3) $T($ If $)=\phi T(f) \forall f \in H(U)$.

Then for each $f \in H(U)$,

$$
T f= \begin{cases}(\bar{f} \circ \bar{\phi}) T(1) & \text { on } W \cap \bar{\phi}^{-1}(U), \\ 0 & \text { on a neighborhood of } W \backslash \bar{\phi}^{-1}(U) .\end{cases}
$$

Proof. Only routine (notational) changes are needed in the preceding proof. For example, now (4) should read

$$
T(f)(w)=T[f(\bar{\phi}(w))](w), \quad f \in H(U), w \in \bar{\phi}^{-1}(U \cap N) .
$$

Corollary 1. Let $U, W$ be regions in $\mathbf{C}, \phi$ a nonconstant holomorphic function on $W$, and $T: H(U) \rightarrow H(W)$ a map which satisfies

(1) $T$ is additive,

(2) $T($ if $)=i T(f)\left(\right.$ resp., $(2)^{*} T($ if $\left.)=i T(f)\right) \forall f \in H(U)$,

(3) $T($ If $)=\phi T(f) \forall f \in H(U)$.

Then either $T=0$ or $\phi(W) \subset U($ resp., $\bar{\phi}(W) \subset U)$ and

$$
T(f)=(f \circ \phi) T(1)(\operatorname{resp} ., T(f)=(\bar{f} \circ \bar{\phi}) T(1)) \quad \forall f \in H(U) .
$$

Proof. Suppose (2) holds. Recall that a nonconstant holomorphic function is an open map. If $W \backslash \phi^{-1}(U) \neq \varnothing$, then according to the theorem every function $T(f)$ vanishes in some nonvoid open subset of $W$. Since $T(f)$ is holomorphic in $W$ and $W$ is connected, it follows that $T(f)=0$. Therefore if $T \neq 0$, then $W \backslash \dot{\phi}^{-1}(U)=\varnothing$, $\phi(W) \subset U$ and the asserted form of $T(f)$ is assured by the theorem. If (2)* holds, the proof proceeds similarly via the scholium.

CoROllaRY 2. Let $U, W$ be regions in $\mathbf{C}, \phi$ a nonconstant holomorphic function on $W$ and $T: H(U) \rightarrow H(W)$ a nonzero map such that

(1) $T$ is additive,

(2) $T($ If $)=\phi T(f) \forall f \in H(U)$.

Then exactly one of the following prevails:

(i) $T$ is linear, $\phi(W) \subset U$ and $T f=(f \circ \phi) T(1) \forall f \in H(U)$.

(ii) $T$ is conjugate-linear, $\bar{\phi}(W) \subset U$ and $T f=(\bar{f} \circ \bar{\phi}) T(1) \forall f \in H(U)$. 
(iii) $T$ is neither linear nor conjugate-linear, $\phi(W) \cup \bar{\phi}(W) \subset U$ and there exist nonzero $\psi_{1}, \psi_{2} \in H(W)$ such that

$$
T f=(f \circ \phi) \psi_{1}+(\bar{f} \circ \bar{\phi}) \psi_{2} \quad \forall f \in H(U) .
$$

Proof. Introduce

$$
T_{1}(f)=\frac{1}{2}[T(f)-i T(\text { if })] \quad \text { and } \quad T_{2}(f)=\frac{1}{2}[T(f)+i T(\text { if })], \quad f \in H(U) .
$$

Then $T_{1}$ satisfies hypotheses (1), (2) and (3) of the preceding corollary and $T_{2}$ satisfies hypotheses (1), (2)* and (3). Note that $T=T_{1}+T_{2}$, that $T_{2}=0$ if and only if $T$ is linear and that $T_{1}=0$ if and only if $T$ is conjugate-linear

REMARK. The hypothesis that $\phi$ be an open map in the above results is not superfluous. Indeed, using a Hamel basis for $\mathbf{C}$ over $\mathbf{Q}+i \mathbf{Q}$, we can easily construct a discontinuous $(\mathbf{Q}+i \mathbf{Q})$-linear functional $\theta$ on $\mathbf{C}$. Notice that such a $\theta$ is not C-linear. Let $U=W$ be an open disc containing $1 \in \mathbf{C}$ and define $T: H(U) \rightarrow H(W)$ by

$$
T(f)=\theta(f(1)) \quad \forall f \in H(U) .
$$

Thus each $T(f)$ is a constant function. Evidently $T($ if $)=i T(f)$ and $T($ If $)=T(f)$ $=1 T(f)$ for all $f \in H(U)$. However, $T$ does not admit of the desired representation since $T$ is not $\mathbf{C}$-linear.

4. Applications to homomorphisms and derivations. Consider two regions $\Omega_{1}, \Omega_{2}$ in C. Let $I_{1}, I_{2}$ denote the identity functions on $\Omega_{1}$ and $\Omega_{2}$, respectively.

THEOREM H. If $T: H\left(\Omega_{1}\right) \rightarrow H\left(\Omega_{2}\right)$ is a ring isomorphism, then either

(i) there is a conformal map $\phi$ of $\Omega_{2}$ onto $\Omega_{1}$ such that $T f=f \circ \phi \forall f \in H\left(\Omega_{1}\right)$, or

(ii) there is a conformal map $\phi$ of $\Omega_{2}$ onto $\Omega_{1}^{*}=\left\{\bar{z}: z \in \Omega_{1}\right\}$ such that $T f=\bar{f} \circ \bar{\phi}$ $\forall f \in H\left(\Omega_{1}\right)$.

Proof. We have $(T i)^{2}=T\left(i^{2}\right)=-T 1=-1$, so that either $T i=i$ or $T i=-i$, because $\Omega_{2}$ is connected. Therefore from the multiplicative property of $T$

$$
\begin{gathered}
\text { either } T(\text { if })=i T(f) \quad \forall f \in H\left(\Omega_{1}\right) \\
\text { or } T(\text { if })=-i T(f) \quad \forall f \in H\left(\Omega_{1}\right) .
\end{gathered}
$$

In the first case

$$
T(\alpha f)=\alpha T(f) \quad \forall f \in H\left(\Omega_{1}\right), \alpha \in \mathbf{Q}+i \mathbf{Q}
$$

and in the second case

$$
T(\alpha f)=\bar{\alpha} T(f) \quad \forall f \in H\left(\Omega_{1}\right), \alpha \in \mathbf{Q}+i \mathbf{Q} .
$$

It follows that the function $\phi=T\left(I_{1}\right) \in H\left(\Omega_{2}\right)$ is not constant. For if $\phi=c$ and we choose $\alpha \in(\mathbf{Q}+i \mathbf{Q}) \cap \Omega_{1} \backslash\{c, \bar{c}\}$, then $\left\{T\left(I_{1}\right)-\alpha 1, T\left(I_{1}\right)-\bar{\alpha} l\right\}$ consists of invertible elements of $H\left(\Omega_{2}\right)$, so $T^{-1}\left\{T\left(I_{1}\right)-\alpha \mathrm{l}, T\left(I_{1}\right)-\bar{\alpha} \mathrm{l}\right\}=\left\{I_{1}-\alpha \mathrm{l}, I_{1}-\bar{\alpha} \mathrm{l}\right\}$ consists of invertible elements of $H\left(\Omega_{1}\right)$, contrary to the fact that $I_{1}-\alpha 1$ has a zero at $\alpha \in \Omega_{1}$. Notice that

$$
T\left(I_{1} f\right)=T\left(I_{1}\right) T(f)=\phi T(f) \quad \forall f \in H\left(\Omega_{1}\right) .
$$


Therefore $T$ fulfills the hypotheses of Corollary 1 . Since $T \neq 0$, it follows that either $\phi\left(\Omega_{2}\right) \subset \Omega_{1}$ and

$$
T f=(f \circ \phi) T(1)=f \circ \phi \quad \forall f \in H\left(\Omega_{1}\right)
$$

or $\phi\left(\Omega_{2}\right) \subset \Omega_{1}^{*}$ and

$$
T f=(\bar{f} \circ \bar{\phi}) T(1)=\bar{f} \circ \bar{\phi} \quad \forall f \in H\left(\Omega_{1}\right) .
$$

By considering $T^{-1} T$ and $T T^{-1}$, one checks that $\phi$ is one-to-one and that either $\phi\left(\Omega_{2}\right)=\Omega_{1}$ or $\phi\left(\Omega_{2}\right)=\Omega_{1}^{*}$. This completes the proof.

THeOREM D. Let $U$ be a nonvoid open subset of $\mathbf{C}, \Delta: H(U) \rightarrow C(U)$ a ring derivation, that is, an additive map which satisfies

$$
\Delta(f g)=f \Delta(g)+g \Delta(f) \quad \forall f, g \in H(U) .
$$

Then $\Delta(f)=f^{\prime} \Delta(I)$ for all $f \in H(U)$.

Proof. Define

$$
T(f)=\Delta(f)-f^{\prime} \Delta(I), \quad f \in H(U) .
$$

Evidently, $T$ is also a ring derivation and $T(I)=0$. So

$$
T(I f)=I T(f)+f T(I)=I T(f) \quad \forall f \in H(U) .
$$

By the derivation property of $T$ again: $T(1)=T(1 \cdot 1)=1 T(1)+1 T(1)$, so $T(1)=0$. Then, too, $0=T(-1)=T\left(i^{2}\right)=2 i T(i)$, so $T(i)=0$. Consequently,

$$
T(\text { if })=i T(f)+f T(i)=i T(f) \quad \forall f \in H(U) \text {. }
$$

We see at last that $T$ fulfills the hypotheses of the Principal Theorem. Since $T(1)=0$, it follows that $T=0$, which proves the present theorem.

5. Historical remarks. A good source for the history of Theorem $\mathrm{H}$ is Alling (1968). Here is a brief account: Theorem $\mathrm{H}$ is due to Bers (1948). A version for $H^{\infty}$ in which the map is assumed complex-linear was proved by $\mathrm{C}$. Chevalley and $\mathrm{S}$. Kakutani in 1942. See Kakutani (1955). This $H^{\infty}$ theorem was improved in Rudin (1955) to cover ring maps. Extensions to Riemann surfaces were found by Royden (1956) and Nakai (1963). A version of Theorem $\mathrm{H}$ which involves isomorphisms of the fields of meromorphic functions on Riemann surfaces was proved by Iss'sa (1966) (see also Nakai (1975)). Other expositions can be found in Alling (1968) and Richards (1970) and the most recent results in Becker and Zame (1979). The case of plane sets $\Omega_{j}$ which need not be open is examined in Su (1972) and Minda (1976).

Theorem D is due to Becker (1968). The proof of it here extends to other contexts, e.g., that in Watatani (1980). A very general version of Theorem $D$ is to be found in Becker and Zame (1979).

\section{REFERENCES}

1. N. Alling, The valuation theory of meromorphic function fields, Entire Functions and Related Parts of Analysis, Proc. Sympos. Pure Math., vol. 11, Amer. Math. Soc., Providence, R.I., 1968, pp. 8-29. MR 38 4700; Zbl 182, 63

2. K. F. Barth and W. J. Schneider, Entire functions mapping arbitrary countable dense subsets and their complements onto each other, J. London Math. Soc. (2) 4 (1971/72), 482-488. MR 46\#353; Zbl $228 \# 30020$ 
3. J. Becker, A note on derivations of algebras of analytic functions, J. Reine Angew. Math. 297 (1978), 211-213. MR 57\#6489; Zbl 375\#46046

4. J. Becker and W. Zame, Homomorphisms into analytic rings, Amer. J. Math. 101 (1979), 1103-1122. MR 82k: 32042; Zbl 473\# 32003

5. L. Bers, On rings of analytic functions, Bull. Amer. Math. Soc. 54 (1948), 311-315. MR 9, 575; Zbl 32, 203

6. H. Iss'sa, On the meromorphic function field of a Stein variety, Ann. of Math. (2) 83 (1966), 34-46. MR 32\#2613; Zbl 146, 314

7. S. Kakutani, Rings of analytic functions, Lectures on Functions of a Complex Variable (W. Kaplan, ed.), Univ. of Michigan Press, Ann Arbor, 1955, pp. 71-83. MR 16, 1125; Zbl 67, 54

8. W. D. Maurer, Conformal equivalence of countable dense sets, Proc. Amer. Math. Soc. 18 (1967), 269-270. MR 35\#6829; Zbl 189, 362

9. C. D. Minda, Rings of holomorphic and meromorphic functions on subsets of Riemann surfaces, J. Indian Math. Soc. (2) 40 (1976), 75-85. MR 56\# 12283; Zbl 436\#30040.

10. M. Nakai, On rings of analytic functions on Riemann surfaces, Proc. Japan Acad. Ser. A Math. Sci. 39 (1963), 79-84. MR 27\#295; Zbl 113, 58

11. __ Divisors of meromorphic function fields, Proc. Japan Acad. Ser. A Math. Sci. 51 (1975), 507-509. MR 52\#5956; Zbl 368\#46030

12. J. W. Nienhuys and J. G. F. Thiemann, On the existence of entire functions mapping countable dense sets onto each other, Nederl. Akad. Wetensch. Proc. Ser. A79 = Indag. Math. 38 (1976), 331-334. MR 57\#631; Zbl 336\#30009

13. M. Ozawa and H. Mizumoto, On rings of analytic functions, Japanese J. Math. 29 (1959), 114-117. MR 22\#9617; Zbl 98, 279.

14. I. Richards, Axioms for analytic functions, Adv. in Math. 5 (1970), 311-338. MR 55\# 10701; Zbl 205, 136

15. H. L. Royden, Rings of analytic and meromorphic functions, Trans. Amer. Math. Soc. 83 (1956), 269-276. MR 19, 737; Zbl 74, 98

16. W. Rudin, Some theorems on bounded analytic functions, Trans. Amer. Math. Soc. 78 (1955), 333-342; Bull. Amer. Math. Soc. 61 (1955), 543. MR 16, 685; Zbl 64, 312

17. D. Sato and S. Rankin, Entire functions mapping countable dense subsets of the reals onto each other monotonically, Bull. Austral. Math. Soc. 10 (1974), 67-70. MR 49\#10883; Zbl. 275\#30020

18. P. Stäckel, Ueber arithmetische Eigenschaften analytischer Functionen, Math. Ann. 46 (1895), 513-520, Jbuch 26, 426; French transl. (by L. Laugel), Nouvelles Annales de Math. (3) 18 (1899), 53-64, Jbuch 30, 383

19. L. P. Su, Rings of analytic functions on any subset of the complex plane, Pacific J. Math. 42 (1972), 535-538. MR 47\# 2076; Zbl 245\#30038

20. Y. Watatani, Derivations on continuous functions, Proc. Amer. Math. Soc. 79 (1980), 206. MR 81g: 46080; Zbl 437\#46050

Department of Mathematics, Kansas State University, Manhattan, Kansas 66506 